\title{
Mongolia: a health system in transition
}

\author{
S Manaseki
}

Genghis Khan in the 13th century was probably brought up imbibing his mother's early morning urine as a remedy for his childhood episodes of acute respiratory infections. In the cold winter temperatures, childhood respiratory infections are still common, and this as well as other home remedies used today in Mongolia may be a remnant of centuries gone by. As I was repeatedly reminded during my six months there, Mongolia has an ancient culture with much of its tradition unchanged since before the time of Genghis Khan. For example, the traditional Mongolian nomadic tents (gers), the traditional script, and other arts and customs are still similar to those times.

Today, however, Mongolia is not a backward, war seeking remnant of Genghis Khan's era. I was struck by the depth of progress and culture in Mongolians' society and their knowledge of the rest of the world despite our ignorance of theirs.

\section{A sparsely populated land}

Mongolia, a landlocked country six times the size of the United Kingdom, has fewer than 2.2 million people, more than $70 \%$ of whom are under 35 years of age. More than three quarters live in towns and cities, but many of them, at least for a few weeks of the year, join the nomadic lifestyle of the rest of their countryfolk. In 1921 Mongolia embraced communism and within three years became the unofficial "sixteenth Soviet republic." Ten years ago, in a move almost unnoticed by the West, it became one of the first of the Eastern bloc satellites to begin breaking away from Soviet control

Soviet dominance made many Mongolians second

$\begin{array}{ll}\text { Arrowe Park Hospital, } & \text { within three years became the unofficial "sixteenth } \\ \text { Upton, Wirral, Merseyside } & \text { Soviet republic." Ten years ago, in a move almost } \\ \text { L49 5PE } & \text { unnoticed by the West, it became one of the first of the } \\ \text { S Manaseki, senior house } & \text { Eastern bloc satellites to begin breaking away from } \\ \text { officer } & \text { Soviet control. } \\ \text { BMF 1993;307:1609-11 } & \text { Soviet dominance made many Mongolians second }\end{array}$

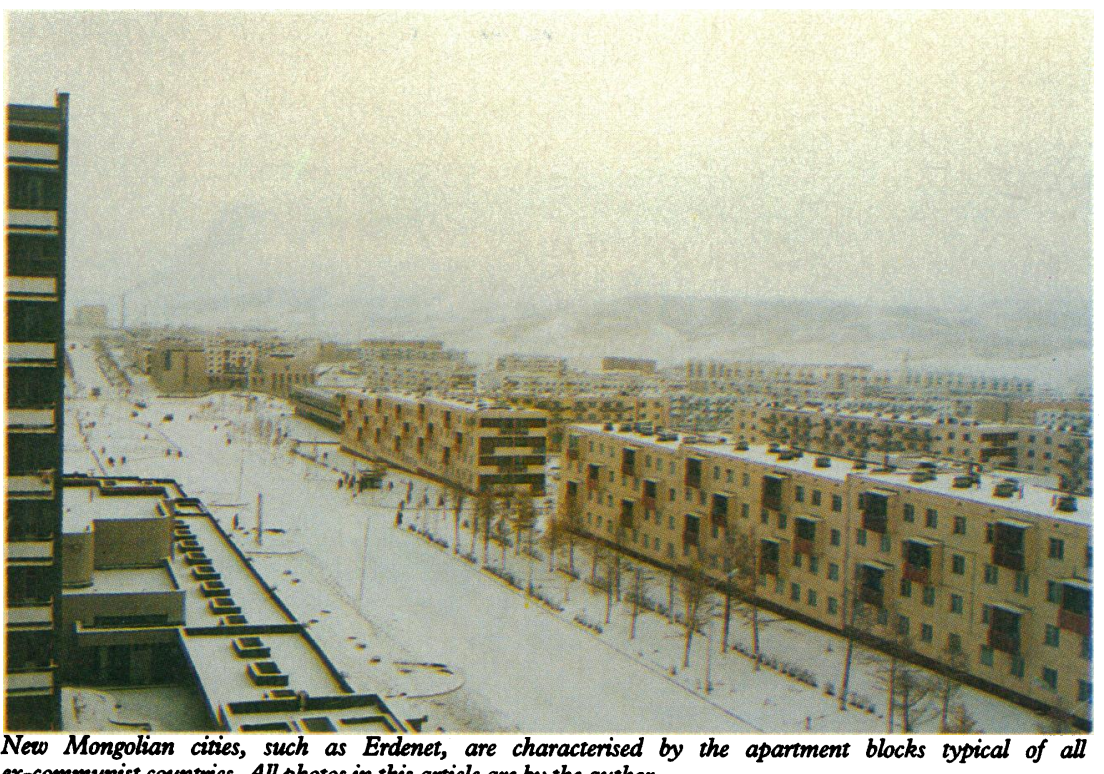
ex-communist countries. All photos in this article are by the author class citizens in their own land and discouraged their traditional culture, religion, language, and arts. However, some positive development also followed. New Mongolian cities and towns are today characterised by highrise apartment blocks as seen in all excommunist countries, with electricity, sanitation, a clean water supply, and effective centralised heating systems. The literacy rate is high, over $95 \%$, especially among women (attendance at secondary school for men is $88 \%$ and for women $96 \%$ ) and, most importantly, a well structured and staffed health care system is accessible to most inhabitants of the country.

In 1990, along with political changes, a market economy was introduced and in January 1991 the Soviet Union, which provided $85 \%$ of development aid to Mongolia, amounting to $35 \%$ of the government's annual budget, discontinued its support. This, along with economic pressures of competing in the world trade, has drastically affected various sectors of society.

Lack of funding has led to limited medical supplies of all kinds, and shortages of fuel and spare parts further limit the distribution of the available resources. Availability of food has also become a problem, and in the past two years ration cards have been the only method of ensuring affordable and adequate quantities of basic foodstuffs to all families. Most basic food items can be found on the black market, but at very high prices - up to 20 times more expensive than with ration cards. Other more luxurious food items, such as vegetables, fruit, and a variety of dairy products, are either not available, particularly in winter months, or are very expensive. Mongolians by tradition prefer a diet of meat and dairy products, and in cases of economic pressure would rather spend on meat than on vegetables. Mongolians are the largest consumers of red meat in Asia (and probably in the world). Fatty meat, if available, will be consumed at all meals, particularly in winter.

Winter is particularly harsh with, temperatures as low as $-40^{\circ} \mathrm{C}$. Winter shortages are worse in the cities, where people rely on supplies from the countryside and abroad. In the countryside each household is more or less self sufficient: nomads obtain meat, milk, and some of their clothing from their animals: they use animal dung and wood for heating and cooking fuel. The more prosperous herdsmen are considered the rich people of Mongolia. "Real Mongolia is in the countryside" was a phrase I heard from many sophisticated city dwellers.

\section{Health system}

Mongolia is a signatory of the Alma Ata declaration of Health for All by the Year 2000, and has tried to develop a well staffed and structured health system to implement the tasks in hand. There are 3.9 doctors for every 1000 people, one of the highest ratios in Asia. More 


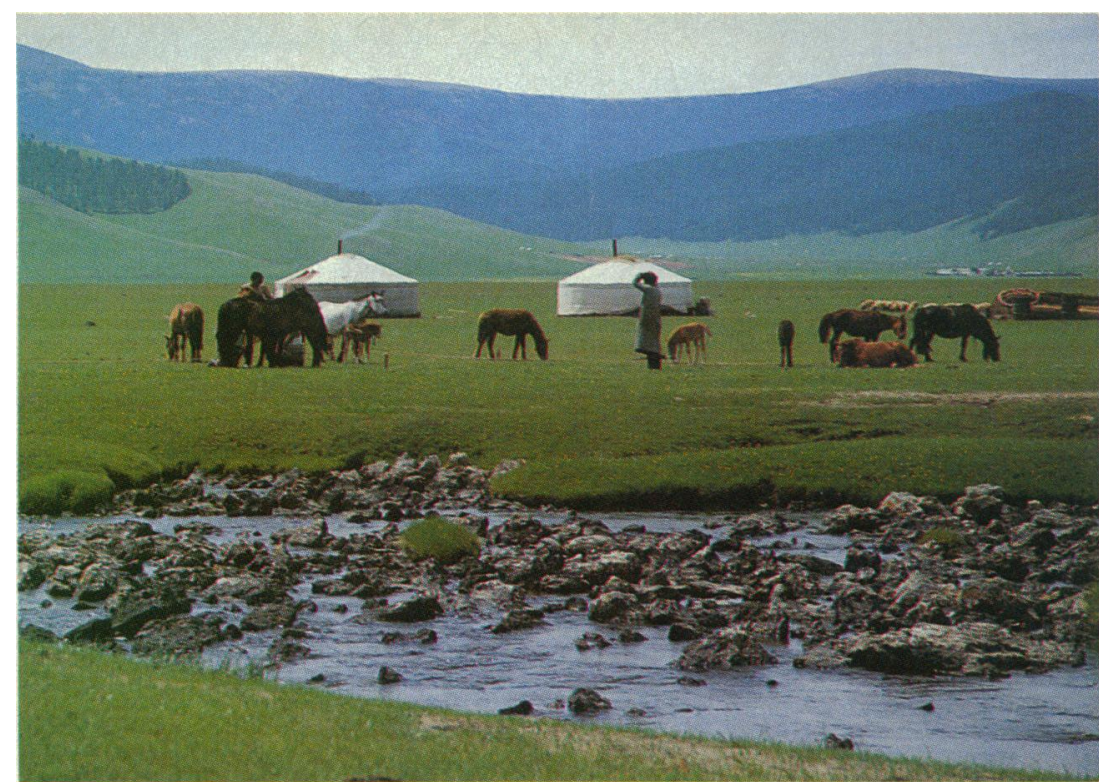

Even city dwellers join the nomadic lifestyle for a few weeks of the year. The steppes, dotted with gers and herdss, conjure great feeling of space

than $80 \%$ of doctors are women, though high positions of authority are often held by their male colleagues. Most doctors return home to cook and look after their families as well as queuing up for a few hours for daily commodities.

The main challenge for health providers is to reach the scattered and constantly moving nomadic population. Acute respiratory infections in the winter and diarrhoeal diseases in the summer are the main cause of mortality and morbidity, especially in children. I could not obtain reliable figures for disease incidence, but I was told by the medical personnel that Mongolians suffer from high rates of hypertension and ischaemic heart disease.

The structure of the health system is currently under review, and most of the country is in the process of changing from a system of centralised specialist clinics to a family doctor system. Erdenet, a new city to the north and the third largest city in Mongolia, was the first to adopt this new system in 1990. "Family doctors" are mostly general physicians, paediatricians, or gynaecologists who had volunteered to become family doctors after attending training seminars. Family doctor clinics consist of three to six doctors, each with a designated nurse, sharing a centre. One doctor is responsible for 200-350 families, including 350-600 children under 16 years. Family doctors are obliged to visit infants under 3 months every two weeks and then every month until 1 year of age. They are also required to visit elderly and homebound chronically ill patients at least once a month. Their nurses accompany them or visit on separate occasions and give injections, change dressings, take baby's measurements, and advise on attending for vaccinations.

Small district hospitals provide care for one or more somons (villages and small towns). They have 10-100 beds and have specialists on staff. At present, the specialty of the doctor seems to make little difference in their appointment in the somon hospitals. For example, in Olzit Horoo, a village near Erdenet, the only two doctors doing all the work in the district ( $>3000$ population) and the 40 bedded hospital were an epidemiologist and a dentist. A third physician, a paediatrician, was on maternity leave, and three others had left to take part in business enterprises. They had joined the ranks of many health care staff, ranging from drivers and nurses to specialist doctors, who have recently left their jobs in order to earn a more manageable wage in the non-medical private sector.
Passing the snuff box is a tradition of Mongolian hospitality
Availability of adequate drugs, raw materials, and functioning equipment for diagnostic tests is a further problem for the doctors in these hospitals.

More fortunate, but still hampered by shortages, are imac (county) hospitals with 250-500 beds and more specialties, which can carry out most routine and some specialist tests. Finally, the few Republic hospitals provide tertiary care in the capital and have full medical and surgical facilities.

Non-conventional therapy in Mongolia is now increasing in popularity, partly because of shortages of conventional medical care. It is divided into two types: treatment without medicine and traditional treatments. Treatment without medicine includes massage, various types of physiotherapy, vacuum cupping, use of ultraviolet and ultrasonic waves and other electromagnetic modalities, focal heat from a light source, iontophoresis, acupuncture, sauna, inhalations, walking on crystals, and other mainly physical treatments. It is available in most hospitals and on a smaller scale at health centres. Traditional treatment is linked to Buddhism and includes herbal remedies as well as religious rituals and the power of prayers. This form of medicine is practised mostly by trained lamas (Buddhist priests). It was banned before 1990 and has become legalised and popularised only recently by a resurgence of Buddhism.

\section{Child health}

Because I was working on projects for Unicef, my attention for much of the time was focused on childhood diseases. Some measures of paediatric primary health care in Mongolia show great achievements. Immunisation coverage has been successful, resulting in the past decade in a reduction in morbidity and mortality from the six diseases covered by the expanded programme of immunisation. Coverage is now $92 \%$ for tuberculosis; $84 \%$ for diphtheria, pertussis, and tetanus; $85 \%$ for polio; and $86 \%$ for measles.

Malnutrition is not a great problem in Mongolia, although vitamin deficiencies, such as of vitamin $D$, certainly are. Almost half of children have at least one sign of rickets (bowed legs or knob knees). One in 40 children under 5 is malnourished; most are newly weaned 1 to 2 years olds who eat with the rest of the family and have inadequate additional food in between family meals. Therefore they have less than the diiet required for growth. All pregnant mothers and those with children under 1 year are entitled to a supplementary ration card for the essentials such as milk, flour, meat, rice, and sugar. "Milk centres" exist in major towns and provide children who have a prescription from the doctor for supplementary free milk and, in some cases, other baby food. Unfortunately this is not always enough either.

Childhood acute respiratory infections are a major problem. In 1988 perinatal mortality was reported to be as high as 12.7 per 1000 live births. Respiratory infections $(28 \cdot 8 / 1000)$ are the commonest cause of death. Infant mortality in 1991 was reported as 62/ 1000 , with more than half due to respiratory infections; pneumonia accounts for about $80 \%$. A problem is that the family doctors have not received adequate training in primary care. Childhood pneumonia is poorly managed by family doctors who were not previously trained as paediatricians.

Despite the high rate of literacy and high physician per population ratio, respiratory infections are often presented to health services too late. Common traditional home treatments include drinking mother's early morning urine (a few teaspoons every few hours); bandaging and bathing with early morning urine or tea, mustard solution, or other substances; and religious rituals and prayers. 


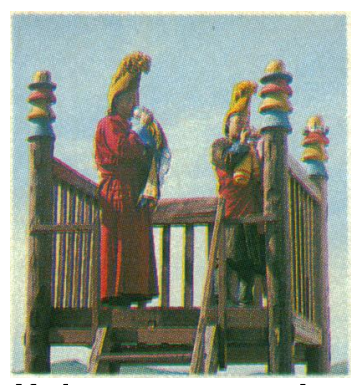

Monks enact a ceremony at the Ganden monastery in Ulan Bator. $A$ resurgence of Buddism has revived interest in traditional treatment
Another possile factor is swaddling. Mongolians tend to swaddle newborn infants from head to toe for the first few months or weeks, exposing only the face, and then from waist down for a few more months. The infant is often swaddled quite tightly with two or three layers of cloth, with or without blankets or a sheep skin, and a rope tied round to keep the child in place. For the first few weeks, every two to five hours the child is unwrapped for only up to half an hour and cleaned. The period of being unwrapped increases as the child grows until swaddling is practised only for sleeping hours.

Keeping the child warm, the excreta in one place and the legs straight are the most popular reasons for swaddling, so swaddling is a form of "nappy" in Mongolia. Sadly, swaddling has been shown to contribute to rickets by blocking the sun's rays. In the countryside a high proportion of the mothers see swaddling as a means of keeping the baby warm in winter, and generally in the cold winter months children are swaddled longer. Literature from other countries reports an increased incidence of pneumonia in swaddled children due to restricted total lung capacity, reduced lung compliance, and the loss of an insulating layer of air around the skin. In Mongolia swaddling is tight and particularly prevalent in winter months; changes in this practice may reduce acute respiratory infections and hence child mortality.

\section{More changes to come}

There is an international recognition that many problems of primary health care programmes are due to lack of knowledge of local practices and lack of sensitivity to economic and cultural factors. Mongolians have a relatively positive view towards conventional medicine. Before the reforms of 1990, traditional medicine had been severely condemned by communism and conventional medical treatment was greatly encouraged. Even now, most people consider a visit to the doctor before or in conjunction with the traditional healer. Public education programmes therefore have some hope of success, especially if local beliefs and practices are taken into consideration.

There is much that the rest of the world can learn from Mongolia, as with many other remote cultures. It was a privilege to be able to have a glimpse of the culture and life of Mongolians at a time of such transformation. Much hardship is created at these periods, and the future is uncertain for many. It will be interesting to observe how, if at all, Mongolia manages to establish a balance between its Eastern roots and Western development, even though it already has a foot in both worlds. In terms of health care, the challenges facing it are shared by many developed and developing countries-the example of Mongolia's response to these challenges could be of benefit to many.

\title{
Travelling as a doctor
}

\author{
Christopher J K Bulstrode
}

When you applied to read medicine, were you asked at interviews why you wanted to read medicine? If so, I wonder what answer you gave. My reason, pure and simple, was that I wanted to travel, and medicine seemed to me a ticket to go anywhere in the world. Somehow I never got round to mentioning that reason at interviews, but still, after all those years of medical training, I was desperate to travel. I was strongly advised against doing preregistration jobs abroad because of the difficulty of getting them recognised. Sadly, I listened to that advice and missed the opportunity of doing a house job in Zimbabwe. But that was the last advice I did listen to. Everyone said that a newly qualified doctor in the Third World is useless. That is true. They then went on to say that you must do an accident and emergency job, an obstetrics and

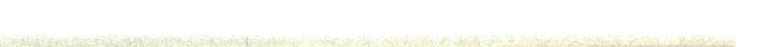

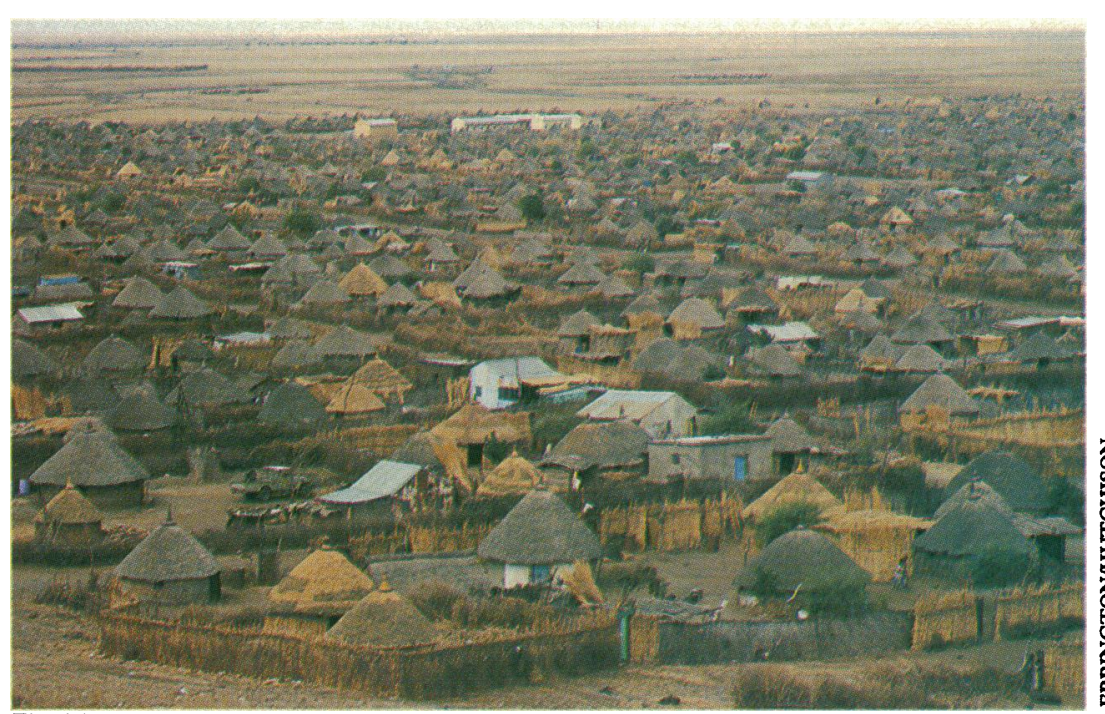

First job overseas: in a Sudanesse refugee camp gynaecology job, a paediatrics job, a surgical job, a dental job, etc, before you are likely to be any use. That is not true. At the end of that set of jobs, not only are you likely to be no use but you are also probably not going to go either. The clutter of responsibilities accumulates rapidly after you qualify, and for most doctors the only time to travel will be straight after registration.

Lesson one: if you have got to travel, do it as soon as possible.

The next problem was who to work for. Missionaries hold a near monopoly of medical jobs in the Third World. Making people better is a cheap and quick way of gaining converts, so medical and surgical clinics are a well recognised annex to many mission stations. My own view is close to that of the Nepalese, who I understand have banned proselytism. I am not religious and have no objections to others being religious but would be obliged if they would keep it to themselves. But a doctor who is not prepared to work at a mission station will find very few openings.

Two of us doctors had decided to team up to provide each other with moral support, and together with my wife we decided to set out freelance and see if we could find work. We had been told that there was a tremendous need for medical care for the Ethiopian refugees in Sudan but had no money to support us. Our first port of call was therefore Geneva, where we visited the various United Nations agencies, each of which is $z$ housed in an extremely tall skyscraper (denoting wealth and power) with an extremely large tree (denoting longevity) in the forecourt. Agencies that provide aid for the Third World may give money but do not want to know what happens to it. The World Council of Churches was sending tens of thousands of pounds each time a disaster occurred, with a promise of hundreds of thousands on receipt of a request for aid, but no effort was made to find out what happened to 